\title{
Dynamic Contact Angle of a Liquid Spreading on a Heated Plate
}

\section{Dean Ripple}

U.S. DEPARTMENT OF COMMERCE

Technology Administration

National Institute of Standards and Technology

Thermophysics Division

Gaithersburg, MD 20899. U.S.A

QC

100

.056

$1 \mathrm{HO} .6351$

1999 



\section{NISTIR 6351}

\section{Dynamic Contact Angle of a Liquid Spreading on a Heated Plate}

\section{Dean Ripple}

U.S. DEPARTMENT OF COMMERCE

Technology Administration

National Institute of Standards and Technology

Thermophysics Division

Gaithersburg, MD 20899, U.S.A

June 1999

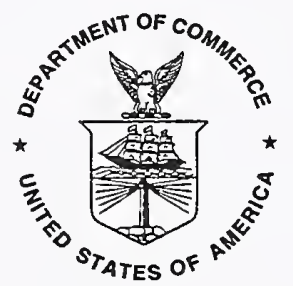

U.S. DEPARTMENT OF COMMERCE William M. Daley, Secretary

TECHNOLOGY ADMINISTRATION Gary R. Bachula, Acting Under Secretary for Technology

NATIONAL INSTITUTE OF STANDARDS

AND TECHNOLOGY

Raymond G. Kammer, Director 



\title{
Dynamic contact angle of a liquid spreading on a heated plate
}

\author{
Dean Ripple \\ Thermophysics Division, \\ National Institute of Standards and Technology, \\ Gaithersburg, MD 20899
}

\begin{abstract}
An equation determining the steady-state profile of a liquid meniscus advancing or receding across a heated plate is derived. The effects of liquid evaporation, intermolecular interaction with the plate, and thermocapillarity are included. Numerical and analytical estimates of the dynamic contact angle are calculated for water and the refrigerant $1,1,1,2$ tetrafluoroethane. The dynamic contact angle depends primarily on three dimensionless parameters: one proportional to the velocity of the interface, one proportional to the rate of evaporation from the surface, and one proportional to the thermal coefficient of the liquid-vapor surface tension.
\end{abstract}




\section{Nomenclature}

(Dimensions in SI units)

English Symbols

$A=$ Hamaker constant in Eq. 1 []]

$a_{1}, a_{2}=$ amplitudes of the solutions to Eq. 25

$b_{1}, b_{2}=$ algebraic combinations of $k_{1}$ and $k_{2}[1 / \mathrm{m}]$

$C=$ constant defined in Eq. $9\left[\mathrm{~kg} /\left(\mathrm{m}^{2} \cdot \mathrm{s} \cdot \mathrm{K}\right)\right]$

$C_{4}, C_{2}, C_{1}, C_{0}=$ constants defined in Eq. 25

$c_{\mathrm{p}}=$ specific heat at constant pressure $[\mathrm{J} /(\mathrm{kg} \cdot \mathrm{K})]$

$D=$ constant defined in Eq. $9[\mathrm{~K} / \mathrm{Pa}]$

$f=$ dimensionless perturbation in a linear expansion of $h(x)$

$g \equiv\left(T_{\mathrm{i}}-T_{\mathrm{v}}\right) / \Theta=$ dimensionless temperature of the liquid-vapor interface

$h=$ dimensionless thickness of the liquid film

$\bar{h}_{0} \equiv \lambda /(C L)=$ natural thermal length used to scale equations $[\mathrm{m}]$

$h_{\infty}=$ dimensionless film thickness in the limit $x \rightarrow-\infty$

$I=$ mass flux of liquid, integrated over film thickness $[\mathrm{kg} /(\mathrm{m} \cdot \mathrm{s})]$

$J_{\mathrm{e}}=$ evaporative mass flux $\left[\mathrm{kg} /\left(\mathrm{m}^{2} \cdot \mathrm{s}\right)\right]$

$k_{1}, k_{2}=$ solutions of Eq. $26[1 / \mathrm{m}]$

$L=$ heat of vaporization of the liquid $[\mathrm{J} / \mathrm{kg}]$

$M=$ molecular weight $[\mathrm{kg} / \mathrm{mol}]$

$m=$ expression defined in Eq. 12

$n=$ number density $\left[1 / \mathrm{m}^{3}\right]$

$p=$ pressure $[\mathrm{Pa}]$

$q_{\mathrm{a}}=$ heat flux by advection through the film $\left[\mathrm{W} / \mathrm{m}^{2}\right]$

$q_{\mathrm{c}}=$ heat flux by conduction through the film $\left[\mathrm{W} / \mathrm{m}^{2}\right]$

$R=$ gas constant $[\mathrm{J} /(\mathrm{mol} \cdot \mathrm{K})]$

$r=$ radius of curvature [m]

$T=$ temperature $[\mathrm{K}]$

$\vec{u}=$ velocity field of the liquid $[\mathrm{m} / \mathrm{s}]$

$\mathrm{v}=$ translational velocity of the interface $[\mathrm{m} / \mathrm{s}]$

$\mathrm{v}_{0} \equiv \gamma /(3 \eta)=$ characteristic velocity used to scale equations $[\mathrm{m} / \mathrm{s}]$

$x=$ dimensionless coordinate along the direction of the interface motion

$y=$ dimensionless coordinate normal to the $x$ and $z$ axes

$z=$ dimensionless coordinate normal to the surface

Greek Symbols

$\alpha=$ evaporation coefficient 
$\beta=$ apparent contact angle of liquid against the solid plate

$\gamma=$ liquid-vapor surface tension $[\mathrm{N} / \mathrm{m}]$

$\delta_{\mathrm{d} 1} \equiv A /\left(\gamma \bar{h}_{0}^{2}\right), \delta_{\mathrm{d} 2} \equiv C D A /\left(v_{0} \vec{h}_{0}^{3}\right)=$ dimensionless intermolecular force parameters

$\delta_{\mathrm{v}} \equiv \mathrm{v} / \mathrm{v}_{0}=$ dimensionless velocity

$\delta_{\Theta} \equiv C \Theta / \mathrm{v}_{0}=$ dimensionless evaporation rate

$\delta_{\mathrm{t}} \equiv-\gamma \Theta /(d \gamma / d T)=$ dimensionless thermocapillary parameter

$\eta=$ dynamic viscosity of the liquid $[\mathrm{Pa} \cdot \mathrm{s}]$

$\Theta \equiv T_{\mathrm{s}}-T_{\mathrm{v}}=$ superheat of the plate surface $[\mathrm{K}]$

$\theta=$ local angle of interface

$\lambda=$ thermal conductivity of the liquid $[\mathrm{W} /(\mathrm{m} \cdot \mathrm{K})]$

$\mu=$ chemical potential [J]

$\Pi=$ disjoining pressure $[\mathrm{Pa}]$

$\rho=$ density $\left[\mathrm{kg} / \mathrm{m}^{3}\right]$

\section{$\underline{\text { Subscripts }}$}

b coordinate where a boundary condition applies

c cut-off length or conduction

co liquid-vapor coexistence

i liquid-vapor interface

1 liquid

s plate surface

$\mathrm{t}$ coordinate translation

$\mathrm{v} \quad$ vapor or velocity

Superscripts

. lengths with physical dimensions.

\section{Introduction}

A variety of liquids, including alcohols and fluorinated refrigerants, completely wet unheated metal surfaces. Boiling of these liquids on a metal surface reveals a strikingly different behavior: the vaporliquid interface of a growing bubble has an apparent non-zero contact angle with the substrate over a wide range of heat fluxes and operating conditions. Many of the theories for boiling processes depend on the vapor-liquid contact angle,[1],[2],[3],[4] but typically this angle must be determined empirically or assumed to be equal to the equilibrium contact angle. The operation of heat pipes also depends on the dynamic contact angle.[5]

The equations for the interaction of a liquid meniscus with a moving surface date back to the work of Landau and Levich.[6] Hervet and de Gennes have extended these ideas to include the effects of intermolecular forces.[7],[8] In this paper, thermal transport, evaporation, and thermocapillary effects are incorporated into the basic equation for the interfacial profile. Throughout the paper 
gravity is neglected and the liquid is assumed to completely wet the solid surface when the surface is not heated. The resulting equation is similar to one derived and discussed by Wayner.[9],[10] Analytic approximations and extensive numerical calculations lead to estimates of the dynamic contact angle and to an understanding of which physical parameters most strongly determine the contact angle.

The equations assume that the contact angles are small and that the thermal transport is dominated by steady state conduction and evaporation. These approximations will introduce errors that are significant for some cases of practical interest. For example, comparison of the solutions to rapidlygrowing vapor bubbles in boiling has limited accuracy because of the short time scale of bubble formation $(10 \mathrm{~ms})$ and the large contact angles $\left(20-40^{\circ}\right)$. The approximations do make the equations more numerically tractable and do not introduce unphysical behavior.

Previous studies on the rapid growth of vapor bubbles during boiling have described the macroscopic development of the bubble size and shape.[4],[11] In this paper, the focus will be on the local shape of the liquid-vapor interface very close to the surface. The equations are intended to be valid only for distances within a few micrometers of the surface of the plate.

Within these limitations, the following results have been found. The contact angle for any fluid can be calculated approximately knowing only the values of three scaled parameters, which depend on the interfacial velocity, the temperature difference between the saturated vapor and the plate, the evaporation rate from the liquid surface, and equilibrium liquid properties. The dynamic contact angle varies only a few percent with an order of magnitude change in the strength of the intermolecular interaction between the plate and the liquid.

The remainder of the report is organized as follows. In Section II, the equations for the interface profile are derived. Section $\mathrm{III}$ describes approximate analytical solutions of these equations. Section IV presents calculations for two specific liquids, water and the refrigerant 1,1,1,2 tetrafluoroethane (R134a), and compares these results with experimental values from the literature. The dependence of dynamic contact angle on various experimental parameters and material properties is also discussed. Section V gives the conclusions.

\section{Equations for the liquid-vapor interface.}

The geometry of the liquid-vapor interface and its contact with the surface of the plate is shown in Fig. 1. At scales of a micrometer or more, the liquid has the appearance of a wedge bounded by solid and vapor. The line where the solid, vapor, and liquid meet is termed the contact line. The slope of the liquid-vapor interface at this line defines the contact angle, $\beta$. On microscopic length scales, this picture is no longer valid. The liquid wedge tapers continuously into a thin adsorbed film of liquid coating the solid surface. Consequently, neither the contact line nor the contact angle are strictly defined. In this report, "contact line" will qualitatively refer to the region where the liquid-vapor 
interface has high curvature. Values for the contact angle will correspond to the slope at a specified point on the interface profile.

The $\bar{z}$ axis is normal to the surface, and the $\bar{x}$ and $\bar{y}$ axes are in the plane of the surface. (Variables with physical dimensions of length are denoted by a bar.) The interface shape is assumed to be in steady state motion, travelling along the plate at a velocity $\mathrm{v}$ along the $+\bar{x}$ direction. The velocity depends in general on the hydrodynamics and thermodynamics of the liquid-vapor interface at large length scales, so $\mathrm{v}$ is an input parameter and not a quantity that is solved for. Let $\bar{h}$ be the thickness of the film, and assume that $\vec{h}$ is independent of the $\bar{y}$ coordinate. The solid plate is assumed to have a fixed temperature, $T_{\mathrm{s}}$, greater than the temperature of the vapor, $T_{\mathrm{v}}$. Gravity will be neglected, and the pressures of the vapor and liquid phases are assumed to be equal far from the contact line of liquid, vapor, and solid phases. Measurements on water suggest that gravity has only a weak effect on the dynamic contact angle.[12]

In the limit $x \rightarrow-\infty$, the film thickness approaches the constant value $\bar{h}_{\infty}$, determined by the competition between evaporation, which favors a dry surface, and the intermolecular forces, which favor a wet surface. The pressure on the liquid-vapor interface resulting from the intermolecular interactions with the plate is termed the disjoining pressure. For a solid-liquid film-vapor geometry, the disjoining pressure $\Pi$ can be approximated in many cases as[13]

$$
\Pi(\bar{h})=\frac{A}{6 \pi \bar{h}^{3}} .
$$

A positive value of the Hamaker constant $A$ corresponds to a pressure favoring a thicker liquid layer. If the surface is hotter than the vapor, the film thickness is stationary provided that the disjoining pressure equals the free energy per unit volume to create liquid out of the adjacent vapor.[14] This free energy can be calculated by making a first order Taylor expansion of the chemical potential of the liquid and vapor phases about the saturation or coexistence temperature, at which the chemical potentials for the two phases are equal. Throughout this paper, $T_{\mathrm{v}}$ will be assumed equal to the coexistence temperature. The resulting equation for $\bar{h}_{\infty}$ is:

$$
\Pi\left(\bar{h}_{\infty}\right)=\left.n_{1}\left[\mu_{1}\left(T_{\mathrm{i}}, p_{\mathrm{i}}\right)-\mu_{\mathrm{v}}\left(T_{\mathrm{i}}, p_{\mathrm{i}}\right)\right] \approx \frac{n_{1}}{n_{\mathrm{v}}} \frac{d p}{d T}\right|_{\mathrm{co}}\left(T_{\mathrm{i}}-T_{\mathrm{v}}\right)
$$

The number densities of the liquid and vapor phases are denoted by $n_{1}$ and $n_{v}$, and $\mu_{1}$ and $\mu_{v}$ are the chemical potentials. Solving this equation gives

$$
\bar{h}_{\infty}=\left[\frac{A}{\left.6 \pi\left(n_{1} / n_{\mathrm{v}}\right)(d p / d T)\right|_{\mathrm{co}}\left(T_{\mathrm{i}}-T_{\mathrm{v}}\right)}\right]^{1 / 3} .
$$

For $T_{\mathrm{i}}-T_{\mathrm{v}} \approx 10 \mathrm{~K}, \bar{h}_{\infty}$ is typically of the order of one monolayer or a few tenths of a nanometer thick. 
The simplest hydrodynamic theories of the liquid flow patterns near the contact line of a liquid, solid, and vapor phase assume that the solid has no adsorbed liquid film coating it and that the velocity of the liquid is zero at the liquid-solid interface. Huh and Scriven[15] demonstrated that these assumptions lead to a frictional dissipation that diverges logarithmically as $\bar{h}(\bar{x}) \rightarrow 0$ unless the no-slip boundary condition is violated. In the present work, the thin film of thickness $\bar{h}_{\infty}$ provides a cut-off for the divergence of the dissipation, and the no-slip condition gives physically reasonable results.

In the limit $x \rightarrow \infty$, the interface shape is determined by the global hydrodynamics of the vapor and liquid phases.[4],[11] The calculations in this paper will assume that the interface has negligible curvature far from the contact line. In certain cases, such as a rapidly-growing vapor bubble, there will be a transition region of high curvature connecting a near-spherical interface in the bulk of the liquid to a film or "microlayer" of liquid coating the surface. The microlayer is akin to a hydrodynamic boundary layer and is typically orders of magnitude thicker than the thin adsorbed film of thickness $\bar{h}_{\infty}$. Applied to physical cases for which microlayers exist, the calculations of this paper describe the liquid-vapor interface only for values of $h$ between $\bar{h}_{\infty}$ and the start of the transition region.

For values of $h$ greater than $\bar{h}_{\infty}$ but much less than any characteristic macroscopic length, the shape of the interface can be described by a set of two coupled equations, one for the mass flow and one for the thermal flow. Provided that the mass flow is sufficiently slow and that the liquid film is sufficiently thin, thermal transport from the surface of the plate to the liquid-vapor interface will take place by conduction through the liquid. If thermal transport from the interface into the vapor is dominated by evaporation, the thermal flux $q_{\mathrm{c}}$ from the interface is proportional to a flux $J_{\mathrm{e}}$ of evaporating molecules. Let $I$ denote the integral over the film thickness of the mass velocity of liquid along the $x$-direction,

$$
I \equiv \int_{0}^{\bar{h}} d \bar{z} \rho_{1} u_{x}(\bar{z}),
$$

where $\vec{u}$ is the velocity field of the liquid and $\rho_{1}$ the liquid density. With $J_{\mathrm{e}}$ measured in units of mass evaporated per unit area per unit time, the equation of continuity for the mass flow in steady state is

$$
\frac{d I}{d x}=-J_{\mathrm{e}}
$$

In the remainder of this section, expressions for $I$ and $J_{\mathrm{e}}$ are derived, and the validity of assuming that conduction dominates the heat transfer is examined.

In the limit $d \bar{h} / d \bar{x}$ « 1 , the thermal conduction is in the $z$ direction and depends only on the local film thickness $\bar{h}(\bar{x})$ and the temperature at the interface, $T_{\mathrm{i}}(\bar{x})$. The heat flux through the film in steady state is given by 


$$
q_{c}=\frac{\left(T_{\mathrm{s}}-T_{\mathrm{i}}\right) \lambda}{\bar{h}}
$$

where $\lambda$ is the thermal conductivity of the liquid.

The evaporative flux from the liquid-vapor interface, $J_{\mathrm{e}}$, can be calculated from kinetic theory.[16] The flux of molecules away from the interface will be equivalent, by detailed balance, to the flux from a volume of vapor at temperature $T_{\mathrm{i}}$ and pressure $p_{\mathrm{i}}$ in equilibrium with the liquid near the interface. The difference in molecular flux between this hot vapor region and the bulk vapor gives the molecular evaporation flux:

$$
J_{\mathrm{e}}=\frac{\alpha\left(p_{\mathrm{i}} / T_{\mathrm{i}}^{1 / 2}-p_{\mathrm{v}} / T_{\mathrm{v}}^{1 / 2}\right)}{\cos \theta(2 \pi R / M)^{1 / 2}} .
$$

In Eq. 7, $\theta$ is the local angle between the $z$ axis and the normal to the $\bar{h}(\bar{x})$ surface, $R$ is the gas constant, and $M$ is the molecular weight. The evaporation coefficient, $\alpha \leq 1$, equals the probability of a molecule from the liquid or vapor phase transferring to the other phase after its path intersects the liquid-vapor interface. Empirically, the value of this coefficient is typically in the range 0.1-1 for organic liquids,[16],[17],[18] but the experimental determinations of $\alpha$ are in general unreliable because $\alpha$ depends strongly on the cleanliness of the liquid surface.

There are three phenomena that effect the evaporation flux: the temperature difference between the interface and vapor, the disjoining pressure, and the Laplace pressure due to the curvature $1 / r$ of the interface. An equation similar to the Kelvin Equation,[19] but with the Laplace pressure replaced by the sum of the Laplace and disjoining pressures, describes the vapor pressure of a curved liquid-vapor interface subject to a disjoining pressure. For $\left|p_{\mathrm{i}}-p_{\mathrm{v}}\right| \ll p_{\mathrm{v}}$ and $\left|T_{\mathrm{i}}-T_{\mathrm{v}}\right| \ll T_{\mathrm{v}}$, a linear expansion in temperature and pressure gives the result

$$
\frac{p_{\mathrm{i}}}{T_{\mathrm{i}}^{1 / 2}}-\frac{p_{\mathrm{v}}}{T_{\mathrm{v}}^{1 / 2}} \approx \frac{1}{T_{\mathrm{v}}^{1 / 2}}\left[\left(\left.\frac{d p}{d T}\right|_{\mathrm{co}}-\frac{p_{\mathrm{v}}}{2 T_{\mathrm{v}}}\right)\left(T_{i}-T_{\mathrm{v}}\right)-\frac{n_{\mathrm{v}}}{n_{l}}\left(\Pi-\frac{\gamma}{r}\right)\right] .
$$

Assuming $d \bar{h} / d \bar{x} \ll 1, r$ can be replaced by $-\left(d^{2} \bar{h} / d \bar{x}^{2}\right)^{-1}$, and the expression for $J_{\mathrm{e}}$ can be simplified by using the approximation $\cos \theta \approx 1$. The resulting equation is

$$
\begin{gathered}
J_{\mathrm{e}} \approx C\left[\left(T_{\mathrm{i}}-T_{\mathrm{v}}\right)-D \Pi(h)-D \gamma \frac{d^{2} \bar{h}}{d x^{2}}\right], \\
C \equiv \frac{\alpha\left[(d p / d T) \|_{\mathrm{co}}-\left(p^{2} / 2 T_{\mathrm{v}}\right)\right]}{\left(2 \pi R T_{\mathrm{v}} / M\right)^{1 / 2}}, \quad D \equiv \frac{n_{\mathrm{v}}}{n_{1}\left[\left.(d p / d T)\right|_{\mathrm{co}}-\left(p \sqrt{\mathrm{v}} / 2 T_{\mathrm{v}}\right)\right]} .
\end{gathered}
$$

The interfacial temperature $T_{\mathrm{i}}$ is determined by the requirement that the heat carried by $J_{\mathrm{e}}$ equal the 
thermal flux $q_{\mathrm{c}}$. Let the superheat $\Theta$ and the function $g(\bar{h})$ be defined as

$$
T_{\mathrm{s}}-T_{\mathrm{v}} \equiv \Theta, \quad T_{\mathrm{i}}-T_{\mathrm{v}} \equiv g(\bar{h}) \Theta .
$$

The heat carried away by the evaporating molecules is $J_{\mathrm{e}}\left(L+c_{\mathrm{p}, \mathrm{v}}\left(T_{\mathrm{i}}-T_{\mathrm{v}}\right)\right)$, where $L$ is the latent heat and $c_{\mathrm{p}, \mathrm{v}}$ is the heat capacity of the vapor. Neglecting the heat capacity term, which is small for typical values of $T_{\mathrm{i}}-T_{v}$, the equation for $T_{\mathrm{i}}$ is

$$
J_{\mathrm{e}} L=\frac{(1-g) \Theta \lambda}{\bar{h}}
$$

Solving for $g$,

$$
g=\frac{1+(\bar{h} / \bar{h}) m}{1+(\bar{h} / \bar{h})}, \bar{h}_{0} \equiv \frac{\lambda}{C L}, m \equiv \frac{D}{\Theta}\left(\Pi+\gamma \frac{d^{2} \bar{h}}{d \bar{x}^{2}}\right) .
$$

For film thicknesses much greater than the natural length scale $\bar{h}_{0}$, the temperature drop across the liquid is a large fraction of the superheat. Consequently, $T_{\mathrm{i}}-T_{\mathrm{v}} \ll \Theta$, and the rate of evaporation is small.

An expression for $I$ can be derived by modeling the hydrodynamics of the liquid film. The flow of liquid through the film is driven by gradients in the pressure of the liquid phase, $p_{1}$, and by gradients in the surface tension, $\gamma$. The vapor phase has a fixed pressure $p_{v}$, so $p_{1}$ at the interface is determined by the pressure difference across the liquid-vapor interface:

$$
\left.p_{1}\right|_{z=h}=p_{\mathrm{v}}+\frac{\gamma}{r}-\Pi \approx p_{\mathrm{v}}-\gamma \frac{d^{2} \bar{h}}{d \bar{x}^{2}}-\Pi
$$

For small $d \bar{h} / d \bar{x}, p_{1}$ can be approximated as a function of $\bar{x}$ only, with $p_{1}(x)=\left.p_{1}\right|_{z=h}$. The velocity field of the flow is found using the lubrication approximation: The flow of liquid induced by gradients in the pressure is approximated by the Poiseulle flow expected for a slab of liquid of the same thickness as the local thickness $\bar{h}(\bar{x})$.

Thermocapillary or Marangoni effects result from transverse forces on the liquid-vapor interface caused by temperature-induced variations of the liquid-vapor surface tension. Almost all single component liquids of low molecular weight have surface tensions that decrease with temperature. Consequently, the surface tension is lowest near the contact line, where the temperature approaches $T_{\mathrm{s}}$. The liquid is pulled by the surface tension from the region near the contact line toward the region of higher surface tension, or from left to right in Fig. 1. Intuitively, this flow will result in a higher contact angle. The numerical calculations in Section IV confirm this conclusion. Birikh[20] derived the appropriate boundary condition for a liquid with this additional shear on the interface: 


$$
\left.\frac{\partial u_{x}}{\partial \bar{z}}\right|_{\bar{z}=\bar{h}} \approx \frac{1}{\eta} \frac{d \gamma}{d T} \frac{d T_{\mathrm{i}}}{d \bar{x}}=\frac{\Theta}{\eta} \frac{d \gamma}{d T} \frac{d g}{d \bar{x}}
$$

In Eq. 14, $\vec{u}$ is the velocity of the liquid, and $\eta$ is the viscosity of the liquid. With this boundary condition and the boundary condition of no velocity slip at the surface of the plate, the velocity field is given by:

$$
u_{x}=\frac{\bar{z}^{2}}{2 \eta} \frac{d p_{1}}{d \bar{x}}+\bar{z}\left[\left.\frac{\partial u_{x}}{\partial \bar{z}}\right|_{\bar{z}=\bar{h}}-\frac{\bar{h}}{\eta} \frac{d p_{1}}{d \bar{x}}\right]-\mathrm{v}
$$

The flux of liquid in the $x$ direction is proportional to the integral of $u_{x}(\bar{z})$ :

$$
\frac{I}{\rho_{1}}=-\frac{\vec{h}^{3}}{3 \eta} \frac{d p_{1}}{d \bar{x}}+\frac{\vec{h}^{2} \Theta}{2 \eta} \frac{d \gamma}{d T} \frac{d g}{d \bar{x}}-\bar{h} \mathrm{v} .
$$

Inserting the expression for $I$ into the continuity equation, Eq. 5, gives a single differential equation for the interface profile. It will be convenient to scale all lengths by $\bar{h}_{0}$, to scale velocities by $\mathrm{v}_{0} \equiv \gamma / 3 \eta$, and to introduce the dimensionless parameters $\delta_{\mathrm{v}}, \delta_{\Theta}, \delta_{\mathrm{t}}, \delta_{\mathrm{d} 1}$, and $\delta_{\mathrm{d} 2}$ :

$$
\begin{gathered}
h \bar{h}_{0}=\bar{h}, x \bar{h}_{0}=\bar{x}, \\
\delta_{\mathrm{v}} \equiv \frac{\mathrm{v}}{\mathrm{v}_{0}}, \delta_{\Theta} \equiv \frac{C \Theta}{\rho_{1} \mathrm{v}_{0}}, \delta_{\mathrm{t}} \equiv-\frac{\Theta}{\gamma} \frac{d \gamma}{d T}, \delta_{\mathrm{d} 1} \equiv \frac{A}{\gamma \bar{h}_{0}^{2}}, \delta_{\mathrm{d} 2} \equiv \frac{C D A}{\rho_{1} \mathrm{v}_{0} \bar{h}_{0}^{3}} .
\end{gathered}
$$

The parameters $\delta_{v}, \delta_{\Theta}$, and $\delta_{t}$ are dimensionless measures of interface velocity, evaporation rate, and thermocapillary effects. The magnitude of the disjoining pressure relative to surface tension and evaporation pressures is characterized by $\delta_{\mathrm{d} 1}$ and $\delta_{\mathrm{d} 2}$, respectively. With these definitions, the main equation for the interfacial profile in dimensionless units becomes:

$$
\begin{gathered}
\frac{d}{d x}\left[h^{3} \frac{d^{3} h}{d x^{3}}+\delta_{\mathrm{t}} \frac{3 h^{2}}{2(h+1)}\left(\frac{1-m}{1+h} \frac{d h}{d x}-\frac{d m}{d x} h\right)-\frac{\delta_{\mathrm{d} 1}}{2 \pi h} \frac{d h}{d x}\right] \\
=\delta_{\mathrm{v}} \frac{d h}{d x}-\delta_{\Theta} \frac{1-m}{1+h} \\
m=\frac{\delta_{\mathrm{d} 2}}{6 \pi \delta_{\Theta} h^{3}}+\frac{\delta_{\mathrm{t}}}{\delta_{\Theta}} \frac{d^{2} h}{d x^{2}} .
\end{gathered}
$$

Solving this equation and examining solutions with $d^{2} h / d x^{2} \rightarrow 0$ as $x \rightarrow \infty$ gives the dynamic contact angle. Wayner[9] has derived an equation similar to Eq. 19 and has discussed the behavior of 
solutions to the equation in various limiting cases. In the limit that the superheat is zero, Eq. 19 is identical to the equation solved by Hervet and de Gennes.[7]

Given the velocity field of the liquid and the temperature distribution as a function of $x$ and $z$, the amount of heat advected by the flowing liquid can be estimated. The net amount of heat per unit time carried by the liquid into a slab of height $h(x)$, unit width, and thickness $\Delta x$ is

$$
q_{\mathrm{a}} \Delta x \approx \int_{0}^{h} d z \rho_{1} c_{\mathrm{p}} u_{x} \frac{\partial T}{\partial x} \Delta x .
$$

Provided that $q_{\mathrm{a}}$ « $q_{\mathrm{c}}$ for the numerical solutions, the approximation that thermal transport is dominated by conduction will be valid. The magnitude of $q_{\mathrm{a}}$ relative to $q_{\mathrm{c}}$ is discussed in Section IV for the solutions to Eq. 19.

\section{Approximate solutions.}

The full equation for the interface profile is too complex to solve analytically. With certain approximations, though, it is possible to find an analytical solution.

Experimental determinations of contact angle are typically visual methods with spatial resolution no better than a micrometer. At this length, disjoining pressures are insignificant. Temporarily, the disjoining pressure will be dropped from Eq. 19 and the resulting equation will be solved in the large $h$ limit.

A useful result corresponds to the limit $v \rightarrow 0$. As a vapor bubble grows from a boiling site, the base of the bubble often grows quickly in its initial stages, but grows slowly or not at all just before the detachment of the bubble from the substrate. The limit $\mathrm{v} \rightarrow 0$ then is physically reasonable.

The evaporation rate can be approximated by first noting that for the range of $\delta_{\theta}$ corresponding to the calculations shown in Figs. 2-6, $m$ « 1. A more drastic assumption is that the dynamic contact angle is determined primarily in the region close to the contact line where $h$ « 1 . Evaluating the evaporation rate in the limits $m \ll 1$ and $h \ll 1$ gives $J_{\mathrm{e}} \approx \Theta \lambda /\left(L \bar{h}_{0}\right)$.

With the final assumption that thermocapillary effects are negligible, Eq. 19 can be solved analytically to get:

$$
h(x) \approx\left(4 \delta_{\odot}\right)^{1 / 4} x\left[\ln \left(\frac{x}{x_{c}}\right)\right]^{1 / 4}, \quad x \gg x_{c} .
$$

The solution has a form similar to the solution for an advancing meniscus on an unheated plate.[7],[8] In particular Hervet and de Gennes demonstrated that for flow over an unheated plate, the cut-off $x_{\mathrm{c}}$ is proportional to a microscopic length characteristic of the range of the intermolecular forces. I expect a similar result for $x_{\mathrm{c}}$ in Eq. 22. The interface slope from this solution is 


$$
\frac{d h}{d x}=\left(4 \delta_{\Theta} \ln \frac{x}{x_{c}}\right)^{1 / 4}\left(1+\frac{1}{4 \ln \left(x / x_{c}\right)}\right)
$$

The corresponding dynamic contact angle is

$$
\beta=\tan ^{-1}\left(\left.\frac{d h}{d x}\right|_{x_{1}}\right)
$$

where a value for $x_{\mathrm{b}}$ must be specified. For large $x_{\mathrm{b}}, \beta$ depends extremely weakly on $x_{\mathrm{b}}$, so estimates of $\beta$ are possible without knowledge of the exact experimental $x_{b}$ values. Because at large values of $h, J_{\mathrm{e}} \sim 1 / h$, the approximate form for $J_{\mathrm{e}}$ overestimates $T_{\mathrm{i}}$ and the evaporation rate. Therefore, the dynamic contact angle as predicted in Eqs. 23 and 24 is expected to be larger than the value predicted by a full numerical solution of Eq. 19.

The dynamic contact angle is not strictly defined in the case when $\mathrm{v} \neq 0$. Substitution of a solution of the form $h=A x+f$ into Eq. 19 gives $f$ « $A x$ only for $\mathrm{v}=0$ and $\delta_{\mathrm{t}}=0$. The slope then has an asymptotic limit of $A$ for $x \rightarrow \infty$. In all other cases, the slope has logarithmic corrections at large values of $x$.

The equation for the interfacial profile is a fourth-order, non-linear differential equation, so four boundary conditions are necessary to completely determine a solution. The desired solution satisfies the boundary conditions $d^{2} h / d x^{2} \rightarrow 0$ as $x \rightarrow \infty$ and $h \rightarrow h_{\infty}$ as $x \rightarrow-\infty$.

In the limit $x--\infty$, the main equation can be approximated by making a linear expansion of $h(x)$ about $h_{\infty}$. Let $h=h_{\infty}+f(x)$, and substitute this form into Eq. 19 to get:

$$
\begin{gathered}
C_{4} \frac{d^{4} f}{d x^{4}}+C_{2} \frac{d^{2} f}{d x^{2}}+C_{1} \frac{d f}{d x}+C_{0} f=0, \\
C_{4}=1-\frac{3 \delta_{\mathrm{t}} \delta_{\mathrm{d} 2}}{2\left(1+h_{\infty}\right) \delta_{\Theta} \delta_{\mathrm{d} 1}}, C_{2}=-\frac{\delta_{\mathrm{d} 1}}{2 \pi h_{\infty}^{4}}-\frac{\delta_{\mathrm{d} 2}}{\delta_{\mathrm{d} 1} h_{\infty}^{3}\left(1+h_{\infty}\right)}+\frac{3 \delta_{\mathrm{t}} \delta_{\mathrm{d} 2}}{4 \pi \delta_{\Theta} h_{\infty}^{4}\left(1+h_{\infty}\right)}, \\
C_{1}=-\frac{\delta_{\mathrm{v}}}{h_{\infty}^{3}}, C_{0}=\frac{3 \delta_{\Theta}}{h_{\infty}^{4}\left(1+h_{\infty}\right)},
\end{gathered}
$$

This linear homogeneous equation has solutions of the form $f=\sum_{i} a_{i} \exp \left(k_{i} x\right)$, where $k$ satisfies the equation

$$
C_{4} k^{4}+C_{2} k^{2}+C_{1} k+C_{0}=0
$$


In general, $k$ will be complex, and there will be two roots with $\operatorname{Re}(k)>0$, and two roots with $\operatorname{Re}(k)$ $<0$. The boundary condition $h \rightarrow h_{\infty}$ as $x \rightarrow-\infty$ requires setting to zero the coefficients for the terms with $\operatorname{Re}(k)<0$. This leaves two coefficients to vary to satisfy the remaining boundary condition.

If the roots $k$ are real, $f$ can be written as

$$
f=a_{1} \exp \left(k_{1} x\right)+a_{2} \exp \left(k_{2} x\right) .
$$

If the roots $k$ are imaginary, $f$ can be written as

$$
f=a_{1} \exp \left(b_{1} x\right) \cos \left(b_{2} x+a_{2}\right),
$$

where $b_{1}$ and $b_{2}$ are algebraic combinations of $k_{1}$ and $k_{2}$. In both cases, a translation in $x, x \rightarrow x+x_{t}$, preserves the form of the solution but changes the magnitude of $a_{1}$ and $a_{2}$. Because the shape of the interfacial profile will be independent of the location of the $x$ origin, or equivalently the choice of $x_{t}$ one of the two coefficients can be chosen arbitrarily.

In the following section, the $x--\infty$ analytic solution, Eq. 27 or 28 , is used for all $x<0$, and at $x=$ 0 the analytic values of $h, d h / d x, d^{2} h / d x^{2}$, and $d^{3} h / d x^{3}$ are used as initial conditions for the numeric solution of Eq. 19 out to $x=x_{\mathrm{b}}, x_{\mathrm{b}}$ 》 1. The one independent coefficient of the analytic solution is adjusted so that the interfacial profile determined numerically from $x=0$ to $x=x_{\mathrm{b}}$ satisfies the boundary condition $d^{2} h /\left.d x^{2}\right|_{x_{b}}=0$.

\section{Results}

Figures 2 and 3 present results for the dynamic contact angle of two liquids used in boiling applications: water and the refrigerant 1,1,1,2 tetrafluoroethane (R134a). Water completely wets only very clean metal surfaces, so the calculated results given here for the dynamic contact angle are possibly lower limits. Water was chosen in spite of this complication because its fluid properties differ substantially from those of the fluorocarbon refrigerant and because it is extensively used in practical applications. The saturated vapor pressure for both calculations was fixed at $100 \mathrm{kPa}$.

The $\delta$ parameters were evaluated using literature values for the equilibrium properties of water[21] and using the computer package REFPROP[22] for the properties of R134a. The Hamaker constant, $A$, depends in general on the dielectric response of both the plate and the liquid over a broad frequency range. From Hough and White, a typical magnitude of $A$ for a solid-liquid-vapor geometry is $10^{-20} \mathrm{~J}$, and this value was used for both water and R134a. Table I lists the values of the parameters used for each liquid.

For all of the calculations reported, the perturbation $f(x=0)$ has a magnitude less than $h_{\infty} / 9$. The numeric calculations were done with a Bulirsch-Stoer differential equation routine,[23] with 64 bit precision and an accuracy of $10^{-11}$ in $h$ and all derivatives. Increasing the accuracy to $10^{-13}$ or varying the size of $f(0)$ had less than $0.2 \%$ effect on the dynamic contact angle. Except where noted, $x_{b}=80$. 
The magnitude of the intermolecular forces enters the interfacial profile equation through the parameters $\delta_{\mathrm{d} 1}$ and $\delta_{\mathrm{d} 2}$. To test for the dependence of contact angle on the magnitude of $A$, the contact angles were recalculated for water and R134a using identical $\delta_{\mathrm{d} 1}$ and $\delta_{\mathrm{d} 2}$ values for both liquids. As seen in Table I and Eq. 17, this is equivalent to varying the Hamaker constant by approximately an order of magnitude for each liquid. The resulting contact angles are shown by the dashed lines in Figs. 2 and 3. For these liquids, a change in $A$ by an order of magnitude gives only a 5-7\% change in contact angle at $x_{b}=80$. Separate calculations in which the value of $A$ is increased to $10^{-19} \mathrm{~J}$ support these results. Over the range of parameters shown in Figs. 2 and 3 , the higher value of $A$ gave a $-9 \%$ to $+2 \%$ change in contact angle for $\mathrm{R} 134 \mathrm{a}$ and a $-16 \%$ to $-1 \%$ change for water.

As seen in Figs. 2 and 3, the curves with $v>0$ intersect the $\delta_{\theta}$ axis. If the velocity of the interface exceeds the intercept value corresponding to the given value of $\delta_{\theta}$, the deposition of a liquid layer will exceed the rate at which the layer can evaporate. In the particular case of a vapor bubble growing in a boiling liquid, the vapor bubble will lie above a thin film of liquid if the bubble growth rate is sufficiently high. The formation of these microlayers has previously been described in terms of the hydrodynamics of the complete vapor bubble, but without consideration of the interaction of evaporation and liquid flow within the microlayer.[11] Figures 2 and 3 show a characteristic velocity above which the microlayer will grow. Below this velocity no microlayer will form, and the surface will dry out to the thickness $h_{\infty}$.

Figure 4 shows the long distance behavior of both the interface slope and the ratio of advection heat flux to conduction heat flux, calculated for R134a at a superheat of $1 \mathrm{~K}$. The $d^{2} h / d x^{2}$ boundary condition was imposed at $x_{\mathrm{b}}=160$, or in physical units, $\bar{x}_{b}=6.5 \mu \mathrm{m}$. At large $x$, the slope varies only weakly with $x$. Consistent with the analytic results in Section III, the slope varies least at large $x$ for the curve with $\mathrm{v}=0$. The plot of the heat flux ratio confirms that at sufficiently small $x$ values and sufficiently small interface velocities, conduction of heat through the liquid dominates any heat flow caused by the transverse velocity of the liquid. Advection is significant only for large values of $x$, at which the value of $d h / d x$ varies only slowly with $x$.

The weak dependence of the contact angle on the parameters $\delta_{\mathrm{d} 1}$ and $\delta_{\mathrm{d} 2}$ suggests that the contact angle can be approximately calculated for any liquid with only a knowledge of $\delta_{b} \delta_{\theta}$, and $\delta_{v}$. Figures 5 and 6 present a series of curves that give the contact angle as a function of these three parameters. The $\delta_{\mathrm{d} 1}$ and $\delta_{\mathrm{d} 2}$ values for these figures are the same geometric averages used for the dashed lines in Figs. 2 and 3. For any liquid with $\delta_{\mathrm{d} 1}$ and $\delta_{\mathrm{d} 2}$ values within 2-3 orders of magnitude of the values listed in Table I, interpolation of the curves in Figs. 5 and 6 will give an estimate of the dynamic contact angle. The analytical approximation of Eq. 23 is also shown in Figs. 5 and 6 as the dashed line. The contact angle depends logarithmically on the undetermined cutoff length $x_{\mathrm{c}}$. For an evaporating liquid, there are three natural length scales characteristic of the intermolecular forces: $h_{\infty}$ and two values of $k^{-1}$ that satisfy Eq. 26. The dashed curve in Figs. 5 and 6 arbitrarily uses the largest value of $k^{-1}$ for $x_{c}$. The analytical approximation agrees with the $\delta_{t} / \delta_{\theta}=0$ curve to within $30 \%$, and as expected gives values of $d h / d x$ that are too large. 
The accuracy of the calculations is difficult to estimate. The equations are correct only to first order in the slope $d h / d x$, so certainly there are errors in the dynamic contact angle of order $(d h / d x)^{2}$. For example, the $\cos \theta$ term in Eq. 7 has been set to unity, and this will give an error of a factor of 1.4 at $d h / d x=1$.

Uncertainties in the materials parameters will also lead to uncertainties in the contact angles. The most serious uncertainty is in the evaporation coefficient, which is difficult to measure with even a factor of 10 certainty for such simple liquids as water. Referring to Fig. 5, a factor of 10 decrease in the evaporation coefficient can decrease the contact angle by as much as a factor of 2-3.

Experimental measurements of dynamic contact angle in boiling liquids are difficult to compare with the theoretical results both because there is a great deal of scatter in the experimental results and because the experimental values correspond to a regime where the assumption $d h / d x$ « 1 is not clearly valid. A further difficulty is that the experiments cannot distinguish between a liquid-vapor interface that merges into a microlayer several micrometers thick and an interface that merges into a nominally "dry" solid surface, where the adsorbed liquid has a thickness of only $h_{\infty}$. The theoretical calculations hold only if the contact angle is observed in the region where $h(x)$ thins down to $h_{\infty}$.

For water, measurements of the dynamic contact angle of the interface include the results: $\beta=52^{\circ}$ to $72^{\circ}$ for a superheat of $10 \mathrm{~K},[4] \beta=35^{\circ}$ to $47^{\circ}$ for superheats in the range of $6.2 \mathrm{~K}-9.4 \mathrm{~K},[12]$ and $\beta=30^{\circ}$ to $47^{\circ}$ for superheats in the range of $15 \mathrm{~K}$ to $26 \mathrm{~K}$. [2] The present calculations give results $50-300 \%$ below these values: $\beta=19^{\circ}$ for a superheat of $6 \mathrm{~K}$ and $\beta=30^{\circ}$ for a superheat of $24 \mathrm{~K}$ at zero interface velocity. Han and Griffith [4] also observe larger apparent contact angles for an interface receding at a higher speed, contradicting the theoretical predictions of smaller contact angles for interfaces receding at higher speeds.

The agreement for refrigerants is better. Kedzierski[24] has recently measured the dynamic contact angles of the vapor bubbles formed during boiling of the refrigerants R11 and R123 on quartz. The contact angles measured for many bubbles were found to be $\beta=31^{\circ} \pm 6^{\circ}$ for R11 and $\beta=36^{\circ} \pm 5^{\circ}$ for R123 at superheats of approximately $25 \mathrm{~K}$. These numbers agree within approximately $50 \%$ with the theoretical results for R134a, which has similar fluid properties: $\beta=38^{\circ}$ for a superheat of $12 \mathrm{~K}$, and $\beta=45^{\circ}$ for a superheat of $24 \mathrm{~K}$.

\section{Conclusions}

The calculations of this paper clarify how a wetting liquid can have an apparent non-zero contact angle on a heated plate. The interface shape is determined primarily by three parameters, $\delta_{v}, \delta_{\theta}$, and $\delta_{v}$ proportional to the interface velocity, the evaporation rate, and the thermal coefficient of the surface tension, respectively. At low superheats, the interface velocity term controls the contact angle. At intermediate superheats, the dynamic contact angle is a slowly varying function of superheat. At high superheats, thermocapillary effects may cause a significant rise in the dynamic contact angle if $d \gamma / d T$ is large. The dynamic contact angle depends on the strength of the intermolecular interactions with the substrate only very weakly. 
Theoretical values for the dynamic contact angle agree with experimentally observed values for refrigerants, but not for water. It may be necessary to include the non-wetting of water on most practical boiling surfaces for better agreement between experiment and theory. For the high values of the contact angle seen in boiling liquids, the accuracy of the theory is limited by the assumptions that the interface slope is small and that the thermal transport is in steady state.

The analytical approximation for the contact angle given by Eq. 23 agrees with the numerical calculations to within approximately $30 \%$ for cases where thermocapillary and interface velocity effects are small.

\section{Acknowledgments}

This work was done while I held an NRC fellowship. I thank R.F. Berg, M. R. Moldover, M. Kedzierski, and S. Davis for useful conversations.

\section{References}

1. W. Fritz, Berechnung des Maximalvolumens von Dampfblasen, Phys. Zeit. 36, 379-384 (1935).

2. R. Cole and H. L. Shulman, Bubble departure diameters at subatomospheric pressures, Chemical Engineering Progress Symposium, Series 64, Vol. 62, ed. J. G. Knudsen, p. 616, AIChE, New York (1966).

3. J. J. Lorenz, B. B. Mikic, and W. M. Rohsenow, The effect of surface conditions on boiling characteristics, Proc. of the 5th International Heat Transfer Conference, Tokyo, Vol. 4, p. 35-39 (1974).

4. C. Y. Han and P. Griffith, The mechanism of heat transfer in nucleate pool boiling-part I, Int. J. Heat Mass Transfer 8, 887-904 (1965).

5. M. N. Ivanovskii, V. P. Sorokin, and I. V. Yagodkin, trans. by R. Berman and G. Rice, The Physical Principles of Heat Pipes, p. 18-30, Oxford (1982).

6. L. Landau and B. Levich, Dragging of a liquid by a moving plate, Acta Physiochim. 17, 42-54 (1942).

7. H. Hervet and P. G. de Gennes, Dynamique du moillage: films precurseurs sur solide "sec," $C$. R. Acad. Sci. (Paris), Ser. 2 299, 499-503 (1984).

8. P. G. de Gennes, Wetting: statics and dynamics, Rev. Mod. Phys. 57, 828-863 (1985). 
9. P. C. Wayner, The effect of interfacial mass transport on flow in thin liquid films, Colloids Surf. 52, 71-84 (1991).

10. M. Sujanani and P. C. Wayner, Microcomputer-enhanced optical investigation of transport processes with phase change in near-equilibrium thin liquid films, $J$. Colloid Interface Sci. 143, 472-488 (1991).

11. H. J. Van Ouwerkerk, The rapid growth of a vapour bubble at a liquid-solid interface, Int. J. Heat Mass Transfer 14, 1415-1431 (1971).

12. R. Siegel and E. G. Keshock, Boiling bubble dynamics in saturated water, AIChE Jour. 10, 509-517 (1964).

13. D. B. Hough and L. R. White, The calculation of Hamaker constants from Lifshitz theory with applications to wetting phenomena, Adv. Colloid Interface Sci. 14, 3-41 (1980).

14. M. Schick, Introduction to wetting phenomena, p. 415-497, in Liquids at Interfaces, Les Houches Session XLVIII, ed. J. Charvolin, J. F. Joanny, and J. Zinn-Justin, North-Holland, Amsterdam (1990). See especially Eq. 3-13.

15. C. Huh and L. E. Scriven, Hydrodynamic model of steady movement of a solid/liquid/fluid contact line, J. Colloid Interface Sci. 35, 85-101 (1971).

16. F. E. Jones, Evaporation of Water, Ch. 3. Lewis Publishing, Chelsea, MI (1992).

17. B. Paul, Compilation of evaporation coefficients, A. R. S. Jour. 32, 1321-1328 (1962).

18. G. M. Pound, Selected values of evaporation and condensation coefficients for simple substances, J. Phys. Chem. Ref. Data 1, 135 (1972).

19. J. S. Rowlinson and B. Widom, Molecular Theory of Capillarity, p. 42. Clarendon, Oxford (1982).

20. R. V. Birikh, Thermocapillary convection in a horizontal layer of liquid, J. Appl. Mech. Tech. Phys. 3, $43-44$ (1966), translation of Zh. Prikl. Mekh. Tekh. Fiz. 3, 69-72 (1966).

21. Handbook of Chemistry and Physics, 73rd Ed., ed. D. R. Lide, p. 6-10, 6-14, 6-29, CRC Press, Boca Raton, FL (1992).

22. G. Morrison and M. O. McLinden, "Application of a Hard Sphere Equation of State to Refrigerants and Refrigerant Mixtures," NBS Technical Note 1226, U.S. Govt. Printing Office, Washington, D.C. (1986). REFPROP is the computer code included in NIST Standard Reference Database 23 and is distributed by the Standard Reference Data Program, NIST, Gaithersburg, MD 20899. 
23. W. H. Press, B. P. Flannery, S. A. Teukolsky, and W. T. Vetterling, Numerical Recipes, p. 563, 582. Cambridge (1986).

24. M. A. Kedzierski, Simultaneous visual and calorimetric measurement of R11, R123, and R123/alkylbenzene nucleate flow boiling, NISTIR 4948. NTIS, Springfield, VA (1992). 
Table I. Values of the parameters used in Eq. 18 for Figs. 2-4. The "average" row gives approximate geometric averages of $\delta_{\mathrm{d} 1}$ and $\delta_{\mathrm{d} 2}$ for the two pure fluids.

\begin{tabular}{|c|c|c|c|c|c|c||}
\hline \hline liquid & $\delta_{\mathrm{d} 1}$ & $\delta_{\mathrm{d} 2}$ & $\delta_{\mathrm{t}} / \delta_{\Theta}$ & $\begin{array}{c}\Theta / \delta_{\Theta} \\
(\mathrm{K})\end{array}$ & $\begin{array}{c}\mathrm{v} / \delta_{\mathrm{v}} \\
(\mathrm{m} / \mathrm{s})\end{array}$ & $\begin{array}{c}\bar{h}_{0} \\
(\mathrm{~nm})\end{array}$ \\
\hline \hline water & $1.58 \times 10^{-5}$ & $8.03 \times 10^{-11}$ & 75.3 & $2.29 \times 10^{4}$ & 69.5 & 103. \\
\hline $\mathrm{R} 134 \mathrm{a}$ & $3.82 \times 10^{-4}$ & $8.85 \times 10^{-8}$ & 14.3 & $1.47 \times 10^{3}$ & 13.0 & 40.5 \\
\hline average & $8.0 \times 10^{-5}$ & $9.0 \times 10^{-9}$ & - & - & - & - \\
\hline
\end{tabular}




\section{Figure Captions}

Figure 1. Schematic of a liquid wedge on a heated surface. The equation for the wedge shape is solved analytically for negative $\bar{x}$ and numerically for positive $\bar{x}$. In the limit $\bar{x} \rightarrow-\infty$, the liquid thickness approaches the constant value $\bar{h}_{\infty}$. A positive interface velocity corresponds to a receding contact line moving to the right. The interface shown is a plot of a numerical solution to Eq. 19 for $\mathrm{R} 134 \mathrm{a}$ at zero interface velocity and a superheat of $1 \mathrm{~K}$. The total width of the $x$ axis is $97 \mathrm{~nm}$, and the total height of the $z$ axis is $15 \mathrm{~nm}$.

Figure 2. Interface slope as a function of superheat for water at a saturated vapor pressure of $100 \mathrm{kPa}$. The filled circles indicate values for the slope at $x_{\mathrm{b}}=80$, obtained from numerical solutions of Eq. 19. The solid curves are guides to the eye. The dashed lines correspond to calculations that use identical values for the parameters $\delta_{\mathrm{d} 1}$ and $\delta_{\mathrm{d} 2}$ for both water and R134a.

Figure 3. Interface slope as a function of superheat for R134a at a saturated vapor pressure of $100 \mathrm{kPa}$.

Figure 4. Interface slope and the ratio of advection to conduction heat flows, as a function of $x$. All of the curves are calculated for R134a at a superheat of $1 \mathrm{~K}$ and a vapor pressure of $100 \mathrm{kPa}$. For this figure only, $x_{\mathrm{b}}=160$.

Figure 5. Interface slope as a function of the scaled parameters $\delta_{t} \delta_{\theta}, \delta_{v}$ and $\delta_{\theta}$. The solid curves are smooth curves that pass through the numerically calculated values of the slope at $x_{\mathrm{b}}=80$. The dashed curve is the analytical approximation described by Eq. 23, with the inverse of the cut-off length equal to the smallest root of the linearized equation, Eq. 26.

Figure 6. Magnification of Fig. 5. 


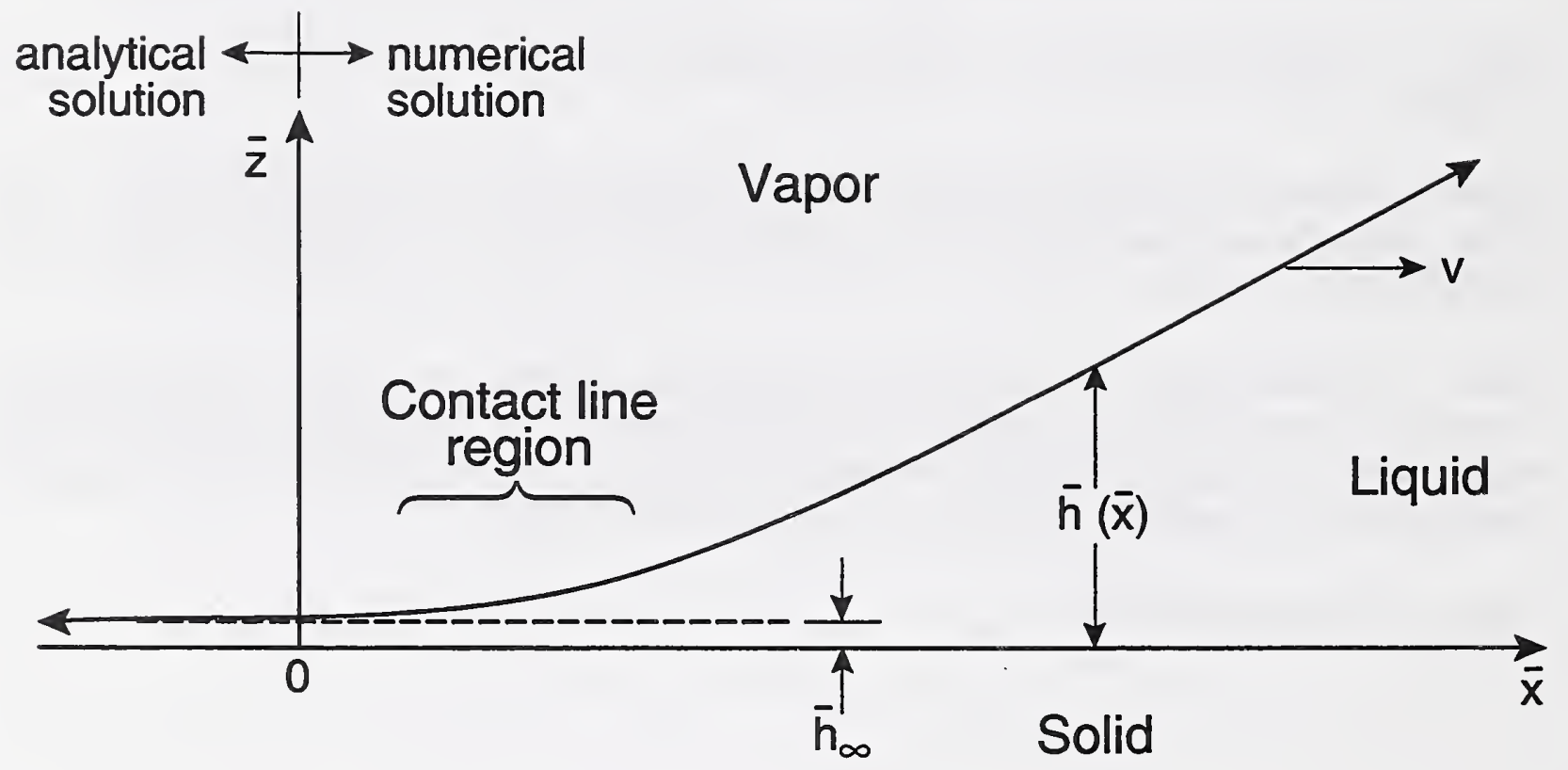

Figure 1 


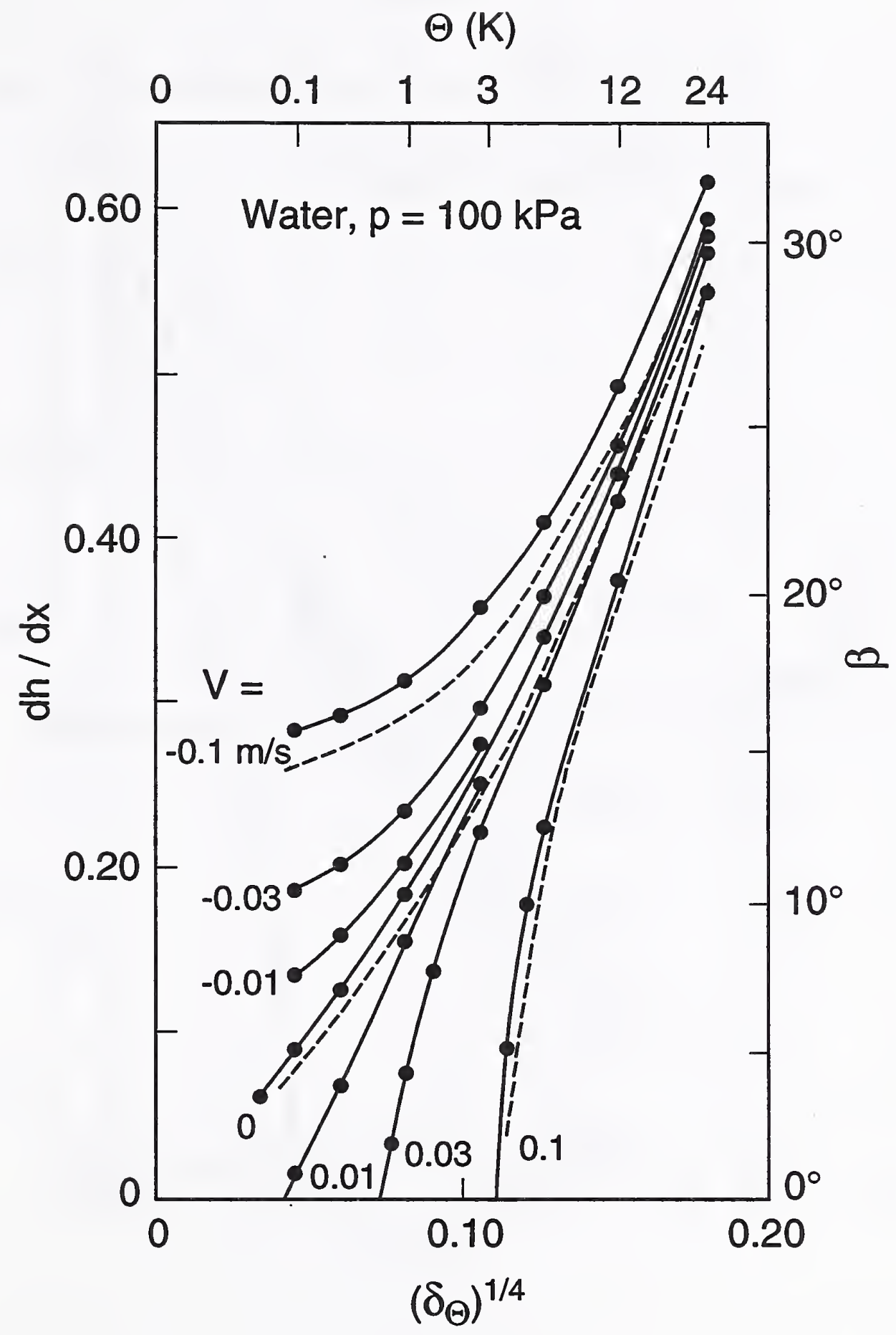

Figure 2 


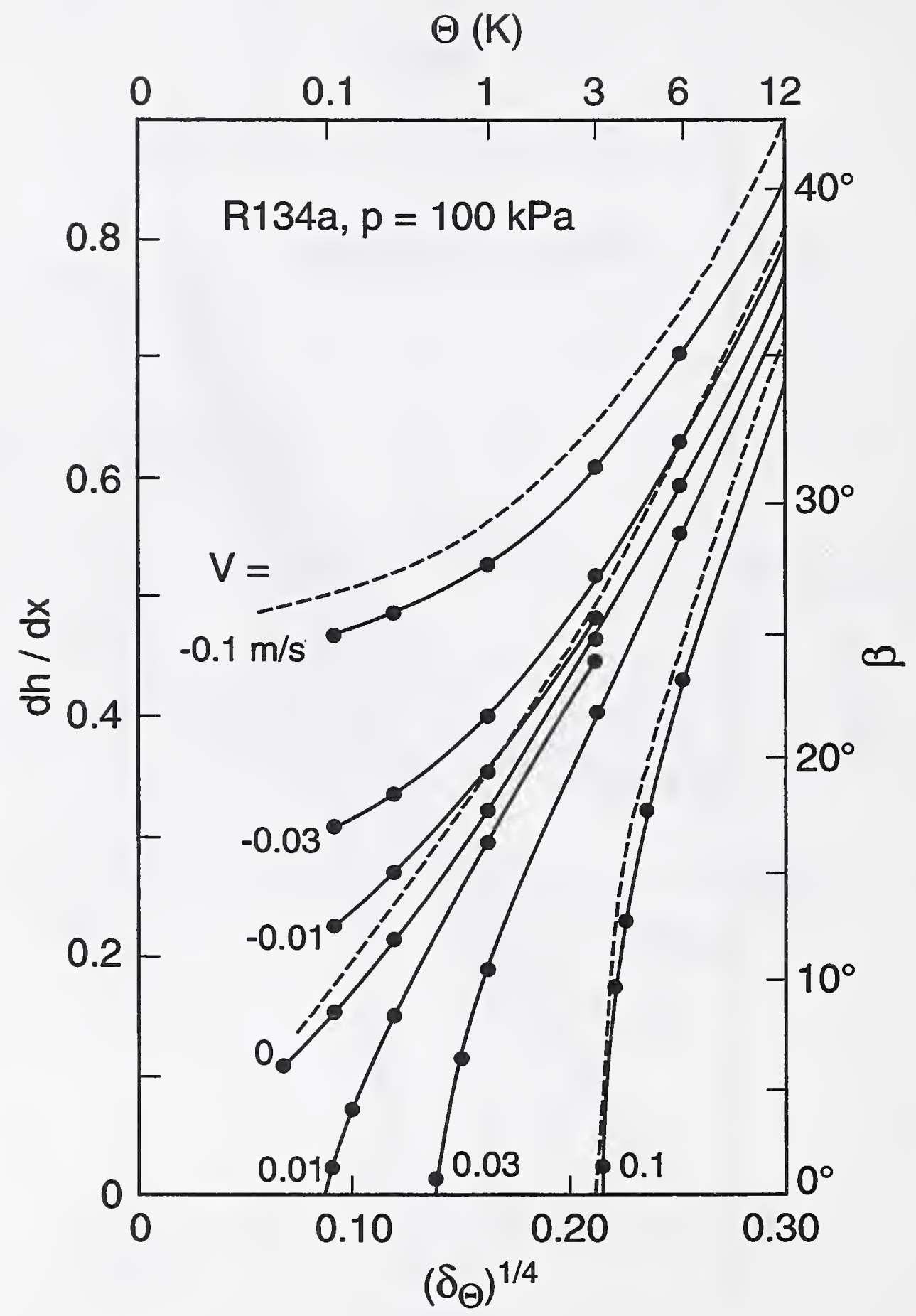

Figure 3 

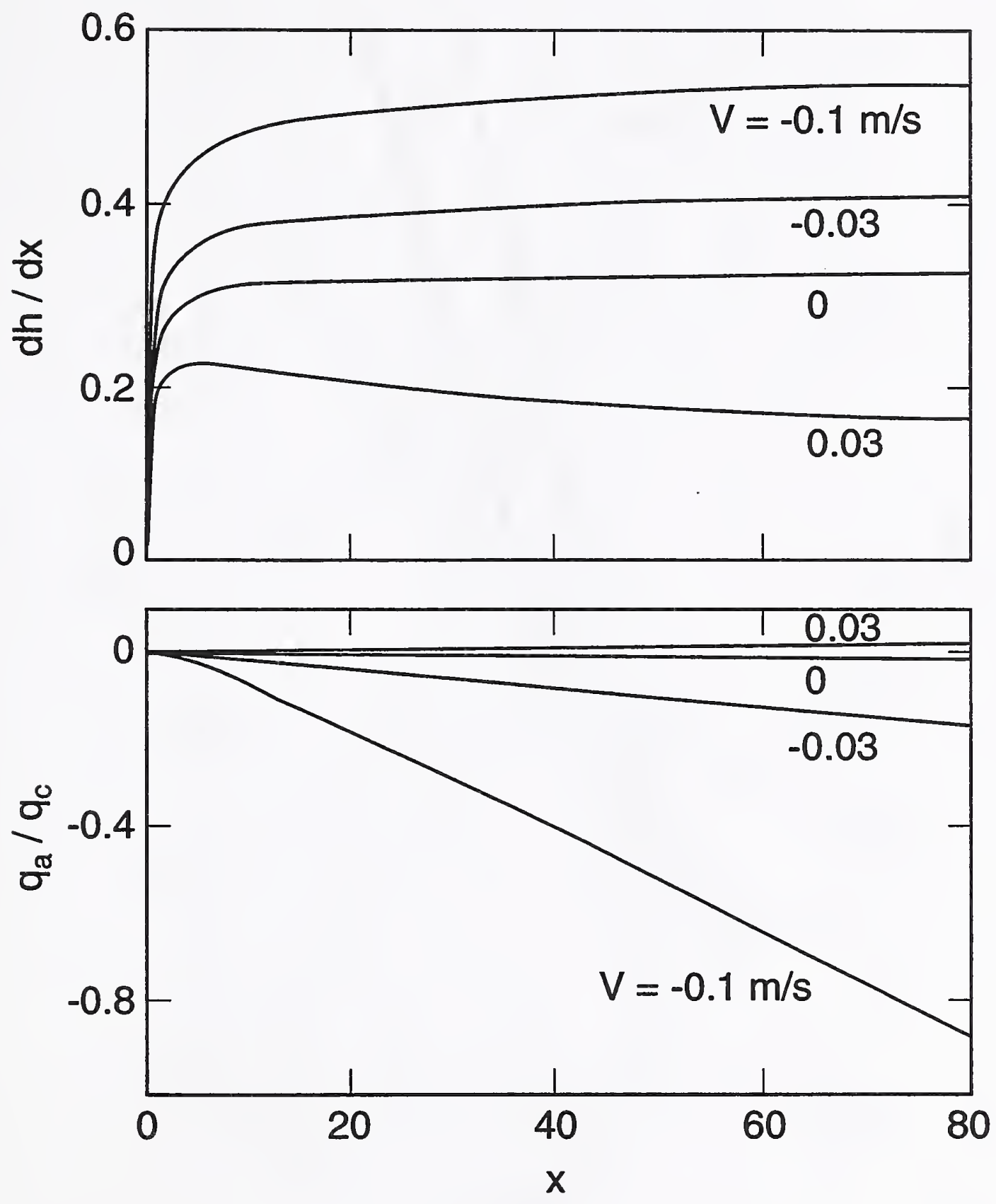

Figure 4 


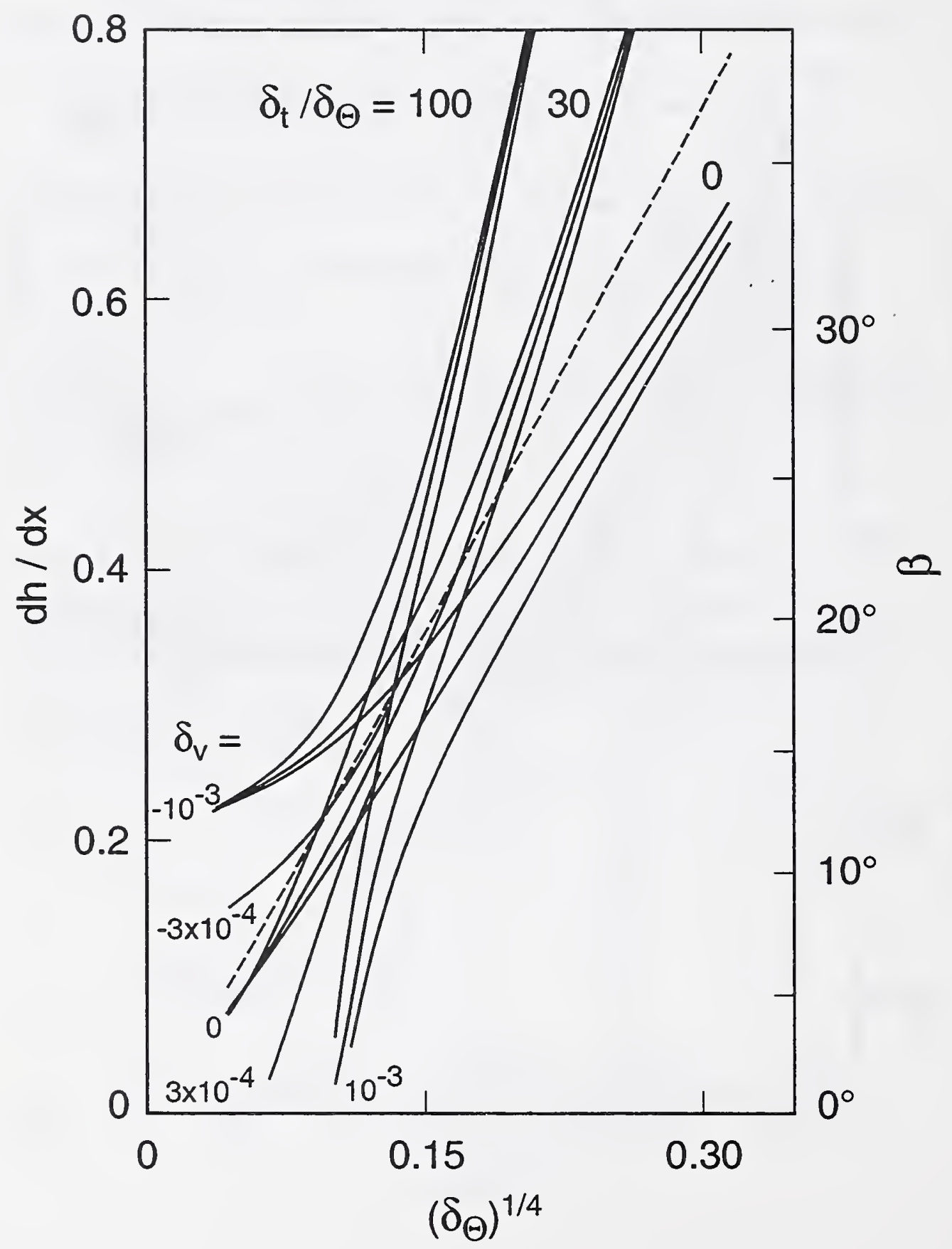

Figure 5 


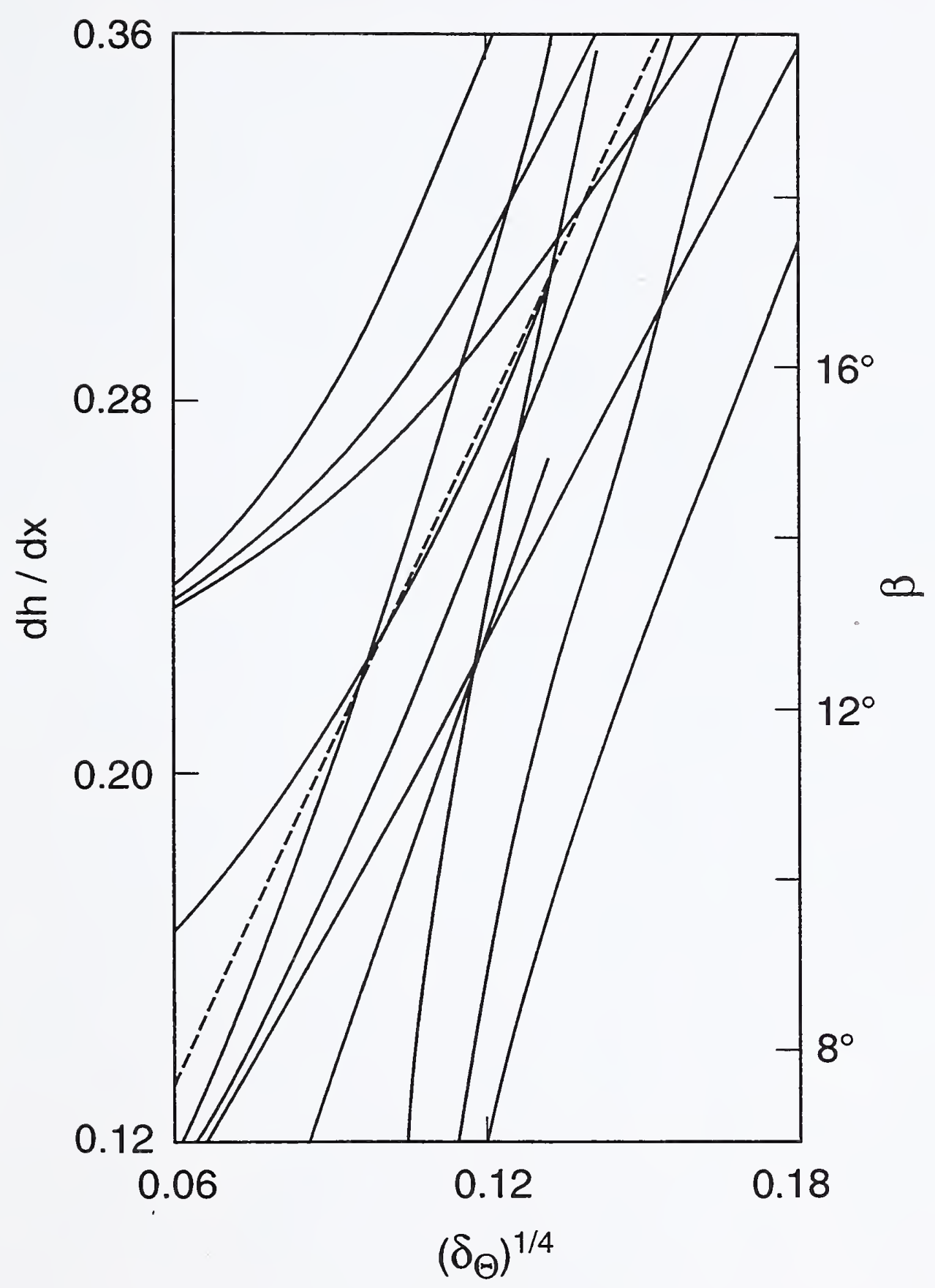

Figure 6 


